\title{
Transgastric and percutaneous transhepatic rendezvous maneuver for the treatment of difficult intrahepatic stones
}

Interventional therapy of hepatic biliary stones is challenging if the patient has been subject to previous gastric or biliary surgery. Several variations have been reported for the endoscopic and endoscopic ultrasound (EUS)-guided transgastric approach to the biliary tract as salvage techniques after the failure of peroral endoscopic drainage [1]. A success rate of $89 \%$ has been reported, with complications seen in $12.5 \%$ of cases [ 2,3$]$.

In the present case, a 62-year-old patient with a Roux-en-Y biliodigestive anastomosis for an intraoperative dissection of the biliary tract that occurred during conventional cholecystectomy presented with progressive jaundice and recurrent colicky pain. Magnetic resonance cholangiopancreatography (MRCP) demonstrated intrahepatic gallstones.

The biliodigestive anastomosis could not be reached by enteroscopy. Therefore percutaneous transhepatic cholangiographic drainage (PTCD) with cholangioscopy was performed for the treatment of the intrahepatic stones. Despite this, persistently elevated levels of alkaline phosphatase and $\gamma$-glutamyltransferase were evident. Repeat MRCP showed a stone-filled, dilated biliary segment in the left hepatic lobe ( Fig. 1) that had not been possible to inspect by cholangioscopy during the PTCD ( Fig. 2) because of complete obstruction of the segmental orifice.

The dilated biliary segment was punctured transgastrically under EUS guidance (GFUE160 linear echoendoscope; Olympus Deutschland GmbH, Hamburg, Germany) using a 19G needle (Cook Echo Tip, Cook Deutschland $\mathrm{GmbH}$, Mönchengladbach, Germany) placed with contrast guidance (ه Fig. 3). A JagWire $(0.035$ inch $\times 450 \mathrm{~cm}$, straight tip; Boston Scientific $\mathrm{GmbH}$, Ratingen, Germany) was inserted into the dilated segment and beyond the stenosis into the main biliary duct. The wire was grasped with a forceps via the percutaneous route by means of cholangioscopy. Balloon dilation ( $6 \mathrm{~mm}$, MaxForce; Boston Scientific $\mathrm{GmbH}$ ) of the segmental stenosis was carried out by the rendezvous procedure. This was followed by laser lithotripsy and complete stone extraction by

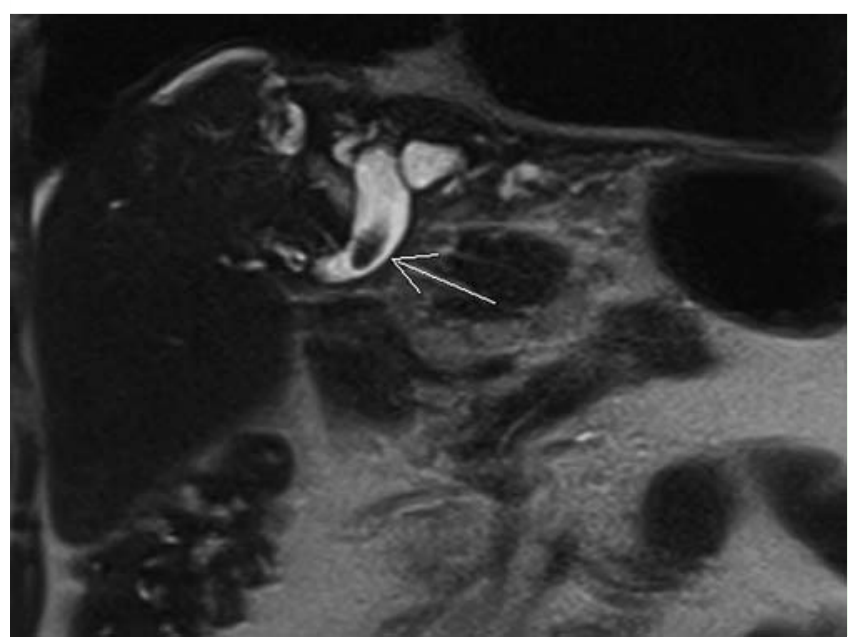

Fig. 1 Magnetic resonance cholangiopancreatography (MRCP) showing biliary stones in a single dilated biliary segment (arrow) of the left hepatic lobe.

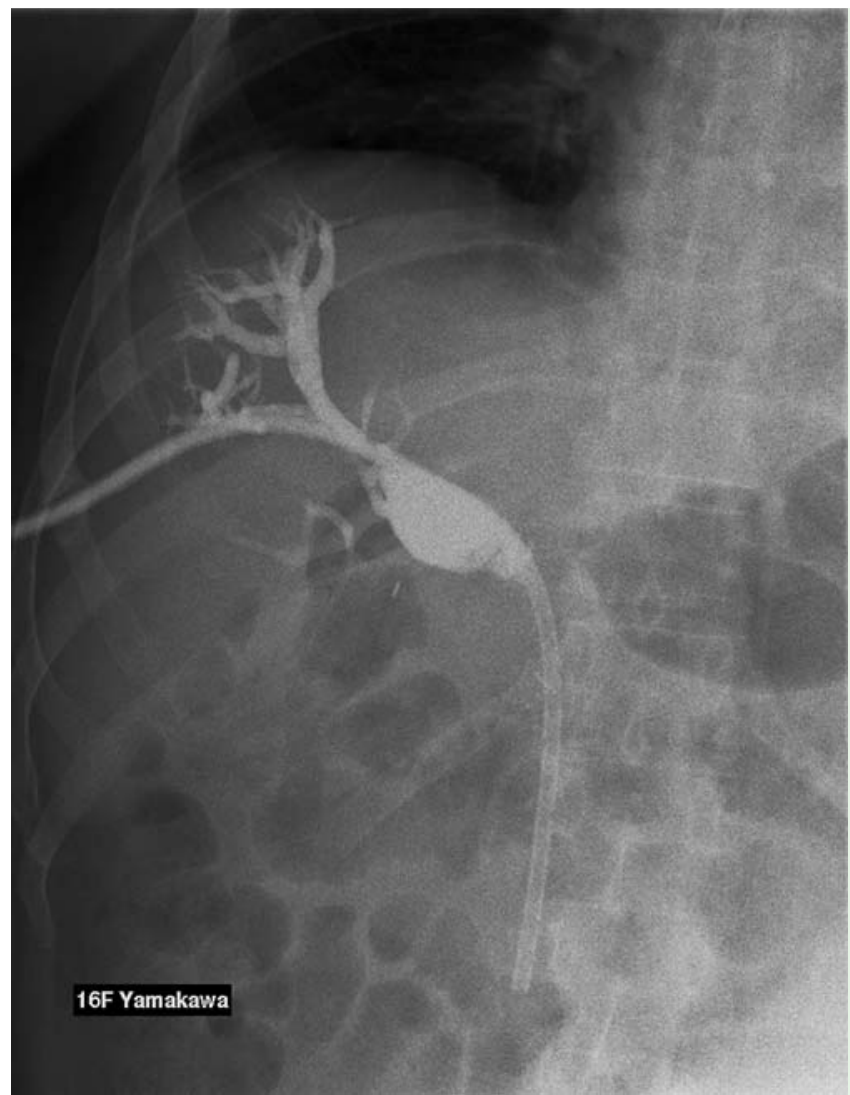

Fig. 2 Percutaneous transhepatic cholangiographic drainage (PTCD) that failed to show the dilated segment later shown on magnetic resonance cholangiopancreatography (MRCP).

basket catheter ( $\bullet$ Fig. 4 ), after which the wire was removed via the transcutaneous route.

In this procedure, not increasing the diameter of the transgastric route to more than the diameter of the needle minimises the risk of biliary leakage, therefore making the transgastric approach to the biliary tract a possible alternative option, even in benign situations.

Endoscopy_UCTN_Code_TTT_1AR_2AH

Competing interests: None 

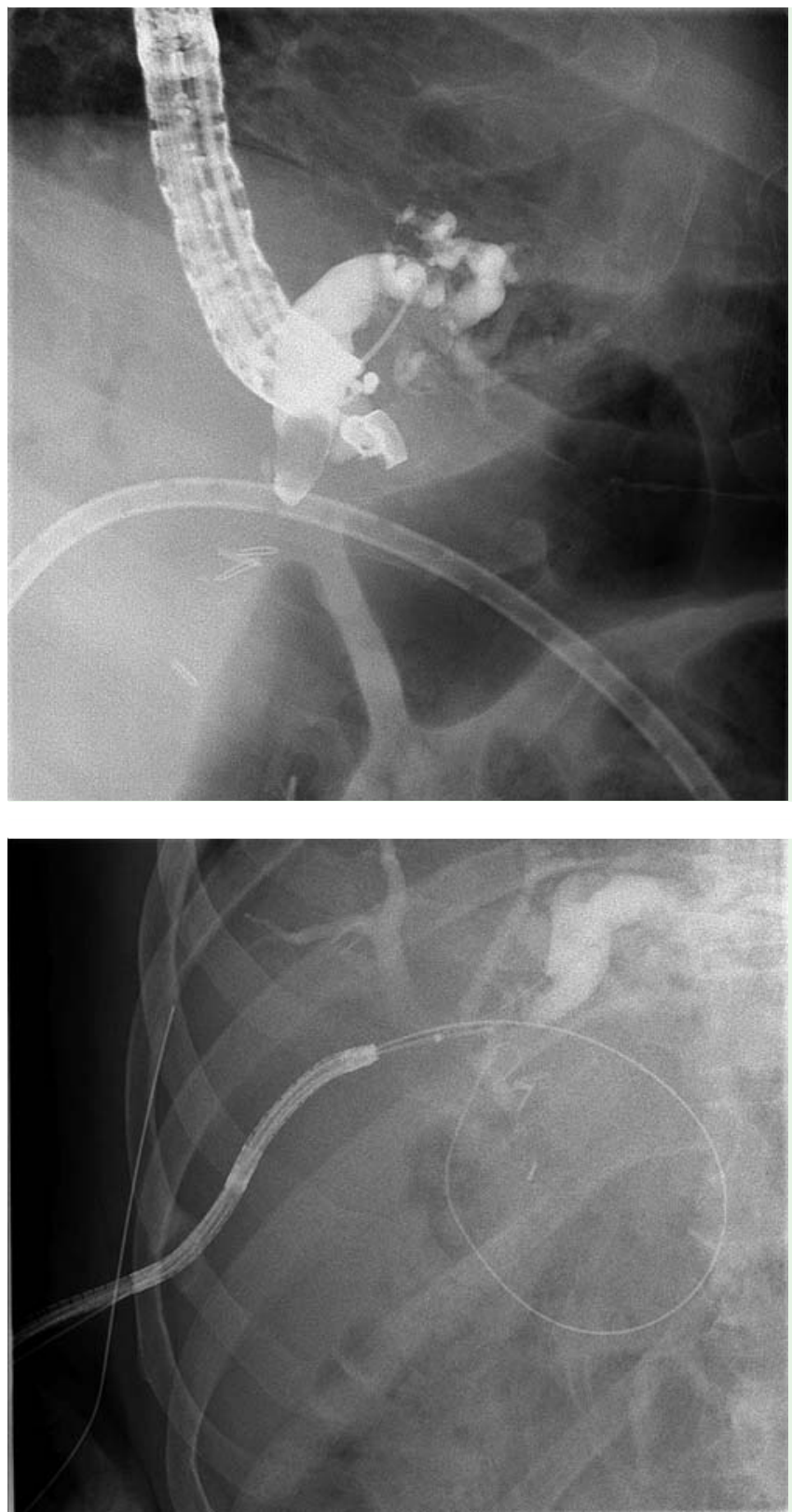

Fig. 3 Endoscopic ultrasound (EUS) image showing the puncture of the dilated biliary segment using contrast guidance.

\section{Erik-Sebastian Fuchs, Bettina Hartmann, Ralf Jakobs}

Med. Klinik C, Klinikum der Stadt Ludwigshafen, Ludwigshafen, Germany

\section{References}

1 Yamao K, Hara K, Mizuno N et al. EUS-guided biliary drainage. Gut Liver 2010; 4: 67-75

2 Bories E, Pesenti C, Caillol F et al. Transgastric endoscopic ultrasonography-guided biliary drainage: results of a pilot study. Endoscopy 2007; 39: 287-291

3 Will $U$, Thieme A, Fueldner $F$ et al. Treatment of biliary obstruction in selected patients by endoscopic ultrasonography (EUS)-guided transluminal biliary drainage. Endoscopy 2007; 39: $292-295$

\section{Bibliography}

DOI http://dx.doi.org/

10.1055/s-0034-1365113

Endoscopy 2014; 46: E184-E185

(C) Georg Thieme Verlag KG

Stuttgart · New York

Fig. 4 PTCD and cholangioscopy showing the formerly obstructed segment now stone free after dilation of the orifice and extraction of the stone.
ISSN 0013-726X

\section{Corresponding author}

\section{Erik-Sebastian Fuchs, MD}

Medizinische Klinik C

Klinikum der Stadt Ludwigshafen gGmbH

Bremserstraße 79

67063 Ludwigshafen

Germany

Fax: +49-621-5034114

fuchse@klilu.de 